\title{
Absorption spectroscopy of adenine, 9-methyladenine, and 2-aminopurine in helium nanodroplets
}

\author{
Szymon Smolarek, ${ }^{a}$ Anouk M. Rijs, ${ }^{b}$ Wybren Jan Buma* ${ }^{* a}$ and Marcel Drabbels ${ }^{* c}$
}

Received 31st May 2010, Accepted 29th June 2010

DOI: $10.1039 / \mathrm{c0cp00746c}$

High-resolution absorption spectra of adenine, 9-methyladenine and 2-aminopurine in helium nanodroplets have been recorded. In contrast to molecular beam experiments, large variations in linewidths are observed for adenine and 9-methyladenine. At the same time, the spectrum of 2-aminopurine remains sharp upon solvation in helium droplets. The line broadening observed for adenine and 9-methyladenine is attributed to a significant decrease of the lifetime of the ${ }^{1} \mathrm{~L}_{\mathrm{b}}\left(\pi \pi^{*}\right)$ state and of ${ }^{1} \mathrm{n} \pi^{*}$ levels vibronically coupled to this state. The origin of the lifetime reduction is argued to be related to the increased accessibility of the ${ }^{1} n \pi^{*} /{ }^{1} \mathrm{~L}_{\mathrm{b}}\left(\pi \pi^{*}\right)$ conical intersection upon solvation of these molecules in liquid helium.

\section{Introduction}

Knowledge about the photophysics and photochemistry of DNA bases is crucial for understanding the carcinogenic potential of UV light. All nucleobases used by nature in the process of replication show very low fluorescence yields and short excited state lifetimes. ${ }^{1,2}$ It is reasonable to conclude that evolution forced this choice in order to prevent deadly effects of UV photochemistry, which could for example lead to function destabilization. While it has become clear by now that the excess electronic energy deposited into DNA bases by the absorption of light is rapidly and efficiently dissipated into vibrational energy, there is still considerable debate on the basic non-radiative relaxation pathways that underlie this dissipation.

The present study concentrates on the nucleobase adenine, its derivative 9-methyladenine, and on 2-aminopurine (Fig. 1). The excited state dynamics of these compounds have been the subject of numerous experimental and theoretical investigations (recent reviews are given in ref. 3-5). The lower-lying excited singlet states of adenine consist of an ${ }^{1} \mathrm{n} \pi *$ state and two states of $\pi \pi^{*}$ character, the so-called ${ }^{1} \mathrm{~L}_{\mathrm{b}}$ and ${ }^{1} \mathrm{~L}_{\mathrm{a}}$ states. ${ }^{6}$ The absorption spectrum of adenine in vapor $^{7}$ and solution phase $^{8,9}$ displays a strong absorption band around $\sim 250 \mathrm{~nm}$. Since the transition moment of the ${ }^{1} \mathrm{~L}_{\mathrm{a}}$ state from the ground state is two orders of magnitude larger than the corresponding transition moments of the ${ }^{1} \mathrm{n} \pi^{*}$ and ${ }^{1} \mathrm{~L}_{\mathrm{b}}$ states, this state is primarily responsible for the absorption observed in this spectral region. Nevertheless, experiments in molecular beams have revealed a number of discrete bands in the $282-272 \mathrm{~nm}$ wavelength region that have been attributed to vibronic transitions to the ${ }^{1} \mathrm{n} \pi^{*}$ and ${ }^{1} \mathrm{~L}_{\mathrm{b}}\left(\pi \pi^{*}\right)$ states. ${ }^{10-16}$ Absorption by the ${ }^{1} \mathrm{~L}_{\mathrm{a}}\left(\pi \pi^{*}\right)$ state is observed in these experiments as well,

\footnotetext{
${ }^{a}$ Van 't Hoff Institute for Molecular Sciences, Faculty of Science, University of Amsterdam, Nieuwe Achtergracht 166,

1018 WV Amsterdam, The Netherlands. E-mail:W.J.Buma@uva.nl

${ }^{b}$ FOM Institute for Plasma Physics Rijnhuizen, Edisonbaan 14 , 3439 MN, The Netherlands

${ }^{c}$ Laboratoire de Chimie Physique Moléculaire, Ecole Polytechnique Fédérale de Lausanne (EPFL), 1015 Lausanne, Switzerland.

E-mail:Marcel.Drabbels@epfl.ch
}

where it manifests itself as a very broad, continuous band without resolved features that starts around $272 \mathrm{~nm}$.

One of the particularly intriguing experimental observations is the large influence the excitation energy appears to have on the excited state dynamics of these molecules. This is exemplified by time-resolved studies of adenine in aqueous solution where the excited state population of the most stable $9 \mathrm{H}$ adenine tautomer has been found to decay with time constants varying from 0.7 to 0.3 ps for excitation wavelengths in the range of 280-245 nm. ${ }^{17}$ Gas phase measurements reveal an even stronger excitation energy dependence. Following excitation of the vibrationless level of the ${ }^{1} \mathrm{~L}_{\mathrm{b}}\left(\pi \pi^{*}\right)$ state, time-resolved photoionization experiments find that the ion signal decays monoexponentially with a time constant of $\sim 9$ ps. ${ }^{11}$ An even longer lifetime of $40-50$ ps has been deduced from fitting the rotational band contour of this spectroscopic feature. ${ }^{14}$ At shorter wavelengths much faster biexponential decays are observed in photoelectron spectra with components of $40 \mathrm{fs}$ and 1.2 ps. $^{3,18-24}$ These results strongly suggest that several channels are of importance for describing electronic relaxation in excited adenine. Indeed, theoretical calculations indicate that various conical intersections are present within the manifold of the electronic ground state $\mathrm{S}_{0}$ and the lower-lying electronically excited $n \pi^{*},{ }^{1} \mathrm{~L}_{\mathrm{b}}\left(\pi \pi^{*}\right)$, and ${ }^{1} \mathrm{~L}_{\mathrm{a}}\left(\pi \pi^{*}\right)$ states, ${ }^{5,25-31}$ while at slightly higher energies also conical intersections with repulsive $\pi \sigma^{*}$ states come into play. ${ }^{31-34}$ It is clear that the decay dynamics will critically depend on the accessibility of these conical intersections and thus on the excitation energy.

High-resolution spectroscopic studies on cold, isolated molecules are appealing starting points to further our understanding of the potential energy surfaces involved in the various decay channels. Besides, it is important to know how these potential energy surfaces - and in particular critical parts like conical intersections - are effected by perturbations induced by interactions with the environment. Helium nanodroplets have emerged in recent years as an almost ideal matrix to reach those goals. ${ }^{35-38}$ Single molecules can be embedded into these droplets and the ultra-low temperature of the droplets $(0.38 \mathrm{~K})$ enables studies at considerably lower internal temperatures of the molecules than possible with 

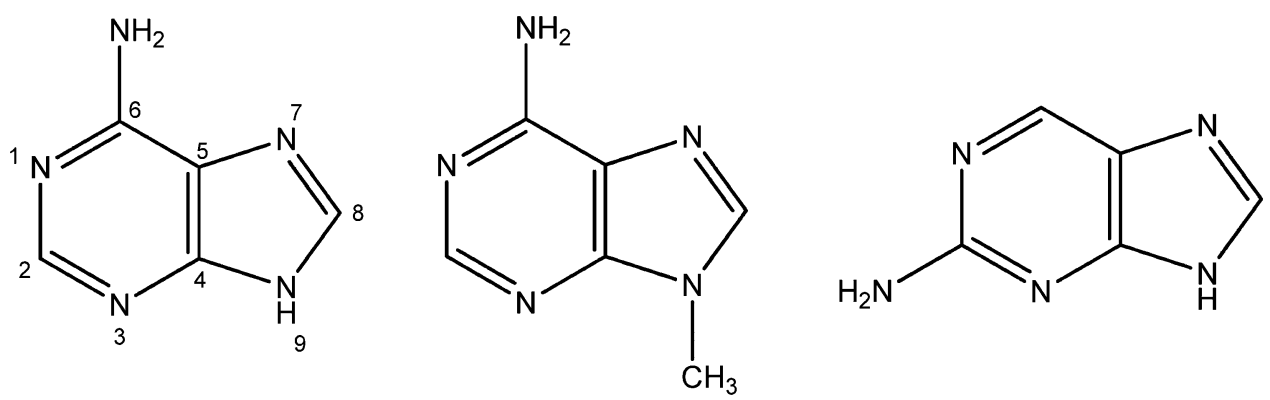

Fig. 1 Chemical structures and atom numbering of adenine (left), 9-methyladenine (middle), and 2-aminopurine (right).

molecular beam techniques. In particular for medium- to large-sized molecules, this lower internal temperature has been found to be crucial to obtain well-resolved spectra. ${ }^{39-42}$ Furthermore, molecules embedded in helium nanodroplets for many purposes can be considered as quasi-isolated, but the helium environment can nevertheless be used to assess the influence of weak intermolecular interactions.

Many high-resolution spectroscopic techniques record in effect excitation spectra, that is, the detected signal is not only determined by the primary absorption process, but also by a secondary process like photon emission or ionization by a second photon. As a result, the detected signal can become critically dependent on the evolution of the molecule in the excited state. For fast, non-radiatively, decaying excited states this can even prevent the recording of spectra. Since helium nanodroplet spectroscopy is generally based on the non-radiative energy transfer from the excited molecule to the helium, ${ }^{43}$ it offers an ideal means to record absorption spectra, especially for those systems exhibiting efficient non-radiative decay pathways.

Here, we present a high-resolution spectroscopic study of adenine, its derivative 9-methyladenine, and of 2-aminopurine (Fig. 1) embedded in liquid helium nanodroplets. Comparison of the absorption spectra recorded under these conditions with excitation spectra in molecular beams shows that the spectroscopy of their excited states is only marginally influenced by the liquid helium matrix, but that their dynamics are modified to a much larger extent. The ramifications of these observations will be discussed in the light of excited-state decay pathways of adenine and solute-solvent interactions.

\section{Experimental methods}

The experimental setup and method used to record electronic absorption spectra of molecules embedded in helium droplets have been described before. ${ }^{44-47}$ In brief, helium droplets consisting of several thousands of atoms are formed by expanding high-purity ${ }^{4} \mathrm{He}$ gas at a pressure of 30 bars into vacuum through a $5 \mu \mathrm{m}$ orifice cooled to cryogenic temperatures by a closed cycle refrigerator. The droplets pick up molecules as they pass through an oven holding the molecule under study. The oven temperature is adjusted for every sample to ensure that the droplets, on average, pick up less than one molecule as they pass through the vapor. For the present experiments, oven temperatures of $100{ }^{\circ} \mathrm{C}, 47{ }^{\circ} \mathrm{C}$, and $220{ }^{\circ} \mathrm{C}$ have been used for adenine, 9-methyladenine, and 2-aminopurine, respectively. The doped droplets enter via a differential pumping stage a fourth vacuum chamber that holds an ion imaging setup. At the center of this setup, the embedded molecules are electronically excited by UV radiation from a $20 \mathrm{~Hz}$ Nd:YAG pumped (Spectra Physics Pro 250) frequency-doubled dye laser (Sirah Precision Scan). The dye laser, operating either on a Rhodamine 610 and Rhodamine 640 mixture (2-aminopurine) or on Pyrromethene 580 (adenine and 9-methyladenine), generates tunable UV radiation with a typical pulse energy of $5 \mathrm{~mJ}$. In our studies on adenine and 9-methyladenine the output of the dye laser was focused by a $300 \mathrm{~mm}$ lens, while in the case of 2-aminopurine an unfocussed beam with a reduced intensity of typically $1 \mathrm{~mJ}$ was used.

Absorption spectra of molecules embedded in helium droplets can be efficiently recorded using evaporation spectroscopy. ${ }^{44}$ This recently developed method relies on the fact that following excitation non-radiative relaxation of the molecules completely evaporates the helium droplets. The bare molecules that remain after evaporation of the droplets are $250 \mathrm{~ns}$ later non-resonantly ionized by the output of an amplified Ti:Sapphire femtosecond laser system (CPA-1000, Clark-MXR) counterpropagating the dye laser beam and focused by a $300 \mathrm{~mm}$ lens. The molecular ions are accelerated by the electric fields of the imaging setup towards a position sensitive detector. By monitoring the number of ions hitting the detector as a function of excitation frequency, absorption spectra can be recorded. As the excited state dynamics of the adenine molecule and its derivates is almost completely governed by non-radiative relaxation processes, this technique is particularly suited for these molecules.

\section{Results and discussion}

An overview of the absorption spectra of adenine, 9-methyladenine and 2-aminopurine in liquid helium nanodroplets is shown in Fig. 2. Before discussing the details of the spectra, it is important to point out that electronic spectra of molecules obtained under such conditions differ in a number of aspects from gas phase spectra. In general, the rovibronic transitions are composed of a narrow zero-phonon line (ZPL) followed by a broad and generally weaker feature, the phonon wing $(\mathrm{PW}){ }^{36,48}$ The zero-phonon line corresponds to excitation of the sample molecule while the broad phonon wing corresponds to simultaneous excitation of the molecule and the surrounding helium. In addition, spectra recorded in helium nanodroplets are usually 
shifted by several tens of wavenumbers compared to gas phase spectra due to two counteracting effects. ${ }^{49}$ Electronic excitation in general leads to an increased polarization of the surrounding helium, which gives rise to a red shift. At the same time, the expansion of the solvation shell leads to a blue shift. Depending on the relative magnitude of these effects, either a blue or a red shift is observed.

Inspection of the spectra presented in Fig. 2 reveals that the overall structure of the spectra in helium droplets is similar to those recorded in the gas phase. ${ }^{10-12,50,51}$ The dominant transitions observed in the gas phase are readily identified in the helium droplet spectra. The limited signal-to-noise ratio of the helium droplet spectra unfortunately prohibits unambiguous identification of transitions with small intensity. While the overall structure of the spectra in helium droplets and in the gas phase is rather similar, clear differences are observed when the widths of the various bands are considered. Many of the rovibronic transitions appear to be significantly broadened upon solvation of the molecules in helium droplets. In order to allow for a quantitative analysis of this effect and an accurate determination of the transition frequencies, we have fitted the lineshapes of the individual vibronic transitions to a Lorentzian line profile. Justification for the use of a Lorentzian profile is provided by the observation that the helium environment induces fast vibrational relaxation, ${ }^{52-56}$ which leads to

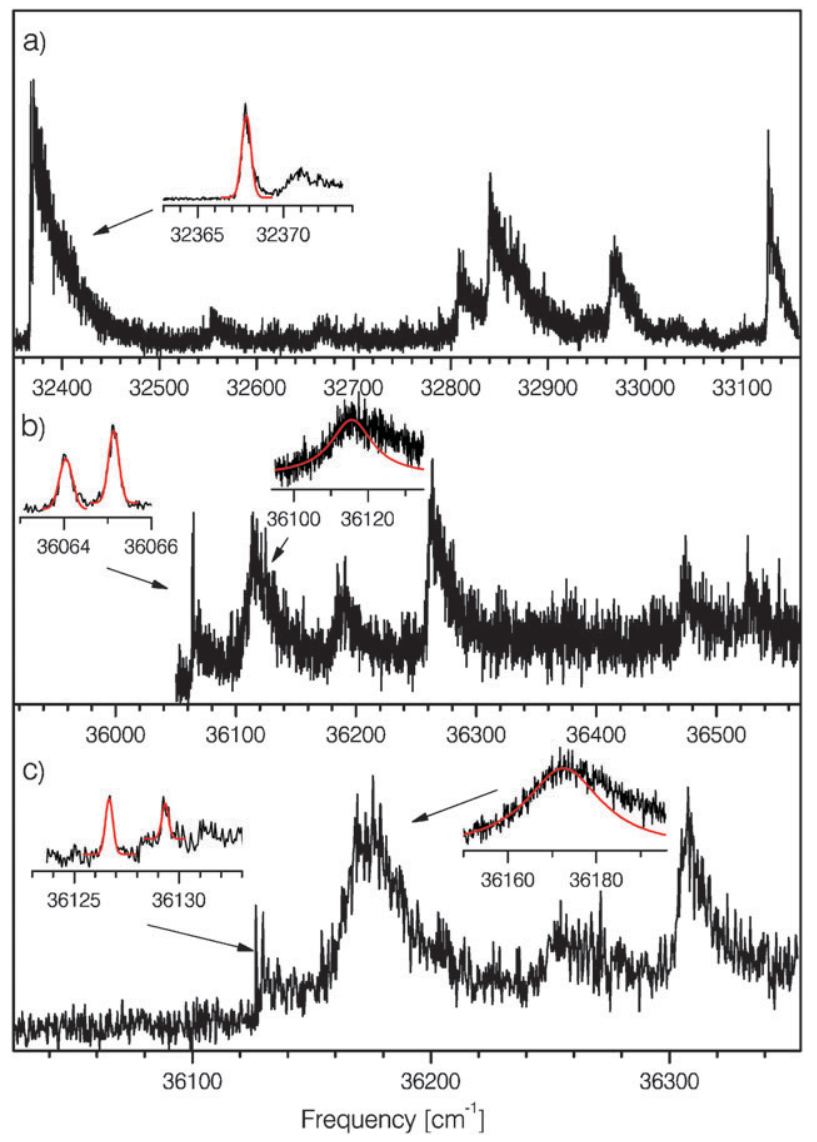

Fig. 2 Absorption spectra of (a) 2-aminopurine, (b) adenine and (c) 9-methyladenine in helium nanodroplets. The insets show expansions of the indicated excitation regions and fits of the bands to a Lorentzian line profile. lifetime broadening of the transitions. To ensure that the resonances were not affected by power broadening, the laser power dependence of each resonance was measured separately. Care was taken that resonance profiles were measured in the regime where the signal intensity was linearly dependent on the laser intensity. For some resonances, the intrinsic linewidth is small enough to observe the ZPL as a feature separated from the PW. In these cases, the complete ZPL was fitted to a Lorentzian profile (see insets Fig. 2). However, the majority of the resonances is significantly broader and has a ZPL that overlaps with the PW. For these resonances only the rising flank, which is determined by the linewidth of the ZPL, was fitted to a Lorentzian profile (see insets Fig. 2). The results of this analysis are presented in Table 1. A comparison between the gas phase and helium droplet transition frequencies reveals that the spectra are shifted - in these cases to the blue-with respect to the gas phase, as is typical for the helium nanodroplet technique. In addition we find a significant variation of the linewidths, especially for adenine and its derivative 9-methyladenine.

Previous experimental REMPI and LIF studies ${ }^{10,51,57}$ as well as theoretical $a b$ initio studies $^{51,58-60}$ on 2-aminopurine have established that the resonances observed in the energy region investigated here are associated with excitation of the ${ }^{1} \mathrm{~L}_{\mathrm{a}}\left(\pi \pi^{*}\right)$ state. Calculations of the potential energy surface of various low-lying electronically excited states show that this state has a stable minimum that lies below the conical intersection of lowest energy, in this case with the ground state. As a result, vibronic levels close to the origin of the ${ }^{1} \mathrm{~L}_{\mathrm{a}}\left(\pi \pi^{*}\right)$

Table 1 Observed frequencies $\left(\mathrm{cm}^{-1}\right)$ and linewidths $\left(\mathrm{cm}^{-1}\right)$ of vibronic transitions of 2-aminopurine, adenine and 9-methyladenine in helium droplets and in the gas phase

\begin{tabular}{lllll}
\hline Label & $\begin{array}{l}\text { Frequency } \\
\text { He droplets }\end{array}$ & $\begin{array}{l}\text { Frequency } \\
\text { Gas phase }\end{array}$ & $\begin{array}{l}\text { Frequency } \\
\text { shift }\end{array}$ & $\begin{array}{l}\text { Linewidth } \\
\text { ZPL }\end{array}$ \\
\hline 2-Aminopurine & & & \\
$P_{1}$ & 32368 & 32368 & 0 & $0.56(2)$ \\
$P_{2}$ & $32556^{d}$ & 32537 & +19 & - \\
$P_{3}$ & $32664^{d}$ & 32643 & +21 & - \\
$P_{4}$ & 32809 & 32802 & +7 & $3.1(4)$ \\
$P_{5}$ & 32841 & 32833 & +8 & $3.3(5)$ \\
$P_{6}$ & 32861 & 32858 & +3 & $2.4(7)$ \\
$P_{7}$ & 32966 & 32963 & +3 & $2.1(4)$ \\
$P_{8}$ & 33127 & 33126 & +1 & $1.26(4)$ \\
Adenine & & & & \\
$A_{1}$ & 36064 & 36063 & +1 & $0.31(2)$ \\
& 36065 & & +2 & $0.25(2)$ \\
$A_{2}$ & 36117 & 36105 & +12 & $12(3)$ \\
$A_{3}$ & 36187 & 36166 & +21 & $7(2)$ \\
$A_{4}$ & 36263 & 36249 & +14 & $3.9(6)$ \\
$A_{5}$ & 36476 & 36463 & +13 & $9(3)$ \\
$A_{6}$ & 36527 & 36519 & +8 & $1.9(9)$ \\
$9-$ Methyladenine & & & \\
$M_{1}$ & 36127 & 36103 & +24 & $0.36(4)$ \\
& 36129 & & +26 & $0.41(9)$ \\
$M_{2}$ & 36173 & 36136 & +37 & $23(4)$ \\
$M_{3}$ & 36255 & 36231 & +24 & $12(3)$ \\
$M_{4}$ & 36306 & 36273 & +33 & $4.1(3)$ \\
$a_{2}$ & & & &
\end{tabular}

${ }^{a}$ Transition frequency in helium nanodroplets. ${ }^{b}$ Transition frequency in the gas phase from ref. 10 for 2-aminopurine and from ref. 11 for adenine and 9-methyladenine. ${ }^{c}$ Obtained by fitting the zero-phonon line to a Lorentzian line shape. ${ }^{d}$ Profiles not fitted, uncertainty in peak position $\sim 5 \mathrm{~cm}^{-1}$. 
transition cannot access barrierless decay channels, and decay on a relatively long time scale. In view of these characteristics of the potential energy surface, one expects that the influence of the helium environment on the electronic spectrum of 2-aminopurine will parallel those observed in previous studies on other molecular systems. This expectation is nicely borne out by the absorption spectrum reported in Fig. 2a, which consists of strong and sharp ZPL's well separated from the accompanying phonon wings. Most bands are blue shifted by roughly the same small amount with the exception of the two very weak bands at 32556 and $32664 \mathrm{~cm}^{-1}$ that show a much larger (blue) shift and appear to be significantly broader than the other transitions. It is interesting to notice that these two particular bands have been assigned to out-of-plane vibrations, while the other bands concern in-plane vibrations. ${ }^{10,61}$ Studies of other molecular systems have revealed a similar difference between in- and out-of-plane vibrational motions, in terms of both line position and linewidth. ${ }^{52,53}$ Apparently, the interaction between the molecule and the surrounding helium environment exhibits a considerable anisotropy for nuclear movement along the different vibrational coordinates. The linewidths of the other transitions in the spectrum of 2-aminopurine follow the general trends as well. The linewidth for the $0-0$ transition is found to be small, $\sim 0.5 \mathrm{~cm}^{-1}$, and is largely determined by the rotational contour at $0.38 \mathrm{~K}^{49}$ The other resonances have slightly larger linewidths. This is attributed to fast vibrational relaxation processes leading to lifetime broadening of these transitions. ${ }^{52-56}$ As in other systems, ${ }^{52,53}$ no systematic variation of the linewidth with excitation energy is observed.

The spectra of adenine and 9-methyladenine in helium nanodroplets (Fig. 2b and c) show much larger deviations from their molecular beam counterparts. This concerns, for example, the absence of resonances in adenine that on the basis of their intensity in the molecular beam spectra would have been expected to be observable (e.g. resonances at 36415 , 36430 , and $36498 \mathrm{~cm}^{-1}$ ). We also notice the absence of the weak feature in the 35500 region that has been attributed to the origin of transition to the ${ }^{1} n \pi^{*}$ state. It appears that this transition is too weak to be detected with the present signal-to-noise ratio. The gas phase spectra of adenine also show resonances in the region of $35825 \mathrm{~cm}^{-1}$ which have been associated with the $7 \mathrm{H}$ tautomer. ${ }^{15}$ Since only the $9 \mathrm{H}$ tautomer of adenine is present in helium droplets ${ }^{62}$ these transitions are absent in our excitation spectra. Even more striking is the variation in the linewidth of the transitions, which is found to vary over two orders of magnitude. This is quite unexpected given that the resonances observed in gas phase studies do not show such large differences. In fact, a rotational band contour analysis of the $A_{1}, A_{2}$, and $A_{4}$ resonances yields Lorentzian linewidths of $\sim 0.1 \mathrm{~cm}^{-1}$ for individual rovibronic transitions. $^{14}$

Although it is generally accepted that the resonances observed in the excitation spectra involve transitions to the ${ }^{1} \mathrm{n} \pi^{*}$ and ${ }^{1} \mathrm{~L}_{\mathrm{b}}\left(\pi \pi^{*}\right)$ states, only a few resonances have been assigned unambiguously. Focusing for the moment on adenine, we notice that historically peak $A_{1}$ has been assigned to a vibronic transition to the ${ }^{1} \mathrm{n} \pi *$ excited state. ${ }^{63,64}$ At first this assignment was only based on estimates of the frequency of the lowest-energy vibration, ${ }^{12}$ but subsequent studies of the dispersed fluorescence ${ }^{13}$ and the rotational band contour ${ }^{14}$ confirmed this conclusion. In helium droplets, this resonance is found to have the smallest width. Actually, the ZPL of this transition is split into two components separated by $2 \mathrm{~cm}^{-1}$ with almost equal intensity and linewidths of $\sim 0.3 \mathrm{~cm}^{-1}$. This width, like in the case of 2-aminopurine, mainly reflects the rotational envelope. Although splitting of ZPL's has been observed in many molecular systems, a complete understanding of the phenomena is still lacking. ${ }^{52,65-67}$ Analysis of the rotational band contour of the $A_{2}$ resonance of gas phase adenine is consistent with a transition involving a ${ }^{1} \pi \pi^{*}$ state, ${ }^{14}$ thus corroborating its previous assignment to the $0-0$ transition to the ${ }^{1} \mathrm{~L}_{\mathrm{b}}\left(\pi \pi^{*}\right)$ state. ${ }^{12}$ In helium droplets, this transition has the largest linewidth, $12.2 \mathrm{~cm}^{-1}$, of the observed transitions. The same gas phase studies concluded that $A_{4}$ involves a transition to the ${ }^{1} \mathrm{n} \pi *$ state as well, albeit as a transition to an out-of-plane vibrational mode which mediates vibronic coupling with the ${ }^{1} \pi \pi^{*}$ state. In our spectra, we observe that the $A_{4}$ transition, corresponding to a level that has a mixed $\mathrm{n} \pi^{*} / \pi \pi^{*}$ character, has a linewidth of $3.9 \mathrm{~cm}^{-1}$, that is, in between those of $A_{1}$ and $A_{2}$. The spectrum of 9-methyladenine, shown in Fig. 2c, reveals the same characteristics as that of adenine. The $M_{1}$ transition, corresponding to the $0-0$ transition to the ${ }^{1} \mathrm{n} \pi^{*}$ state, has a narrow linewidth determined by the rotational contour of the resonance. At the same time, the $M_{2}$ transition corresponding to the band origin of the ${ }^{1} \mathrm{~L}_{\mathrm{b}}\left(\pi \pi^{*}\right) \leftarrow \mathrm{S}_{0}$ has the largest linewidth of all observed transitions in 9-methyladenine. The two other transitions observed, which are thought to be of mixed $n \pi * / \pi \pi^{*}$ character, have linewidths intermediate between these.

The linewidth of the $A_{1}$ transition of adenine in the present study is similar to what was found in the gas phase. However, the $A_{2}$ and $A_{4}$ resonances have considerably larger linewidths. Assuming that this line broadening is the result of fast relaxation, this signifies that the lifetime of the associated levels has decreased upon solvation in helium from more than 50 ps to $\sim 430$ fs and $\sim 1.4 \mathrm{ps}$, respectively. It thus appears that in the helium environment the non-radiative decay of levels of (partial) ${ }^{1} \pi \pi^{*}$ character is significantly increased. Such a conclusion is consistent with the experimental observation that we have not been able to detect resonance-enhanced two-photon ionization signals of adenine in liquid helium droplets, even though energetically the two-photon energy is above the energy of the $\mathrm{D}_{0}\left(\pi^{-1}\right)$ ionic ground state. ${ }^{18,68,69}$ The apparently small cross section for ionization can be understood if adenine excited-state levels with significant ${ }^{1} \pi \pi^{*}$ character decay rapidly to states from which ionization is severely impeded.

As discussed in the introduction, the decay pathways of electronically excited purines, and in particular those of adenine, have been the subject of many experimental and theoretical studies. The general consent is that the decay dynamics are governed by a number of conical intersections involving the electronic ground state and the lower electronically excited ${ }^{1} \mathrm{n} \pi^{*},{ }^{1} \mathrm{~L}_{\mathrm{b}}\left(\pi \pi^{*}\right)$, and ${ }^{1} \mathrm{~L}_{\mathrm{a}}\left(\pi \pi^{*}\right)$ states. At excitation wavelengths of $267 \mathrm{~nm}$ or shorter the ${ }^{1} \mathrm{~L}_{\mathrm{a}}\left(\pi \pi^{*}\right)$ state is the dominantly absorbing state. Non-radiative decay of this state occurs mainly via a conical intersection with 
the ground state that is reached along a barrierless path involving a ring-puckering coordinate of the six-membered ring (out-of-plane rotation of the $\mathrm{C}_{2}-\mathrm{N}_{3}$ bond). ${ }^{5,27-31}$ Other channels that have attracted considerable attention involve conical intersections with repulsive ${ }^{1} \pi \sigma^{*}$ states along the $\mathrm{N}_{9}-\mathrm{H}$ and $\mathrm{N}_{10}-\mathrm{H}$ coordinates. ${ }^{32-34,70-72}$ These channels, however, only open up at wavelengths shorter than $233 \mathrm{~nm}^{71}$ and would thus a priori not be expected to be involved in the decay of the ${ }^{1} \mathrm{n} \pi^{*}$ and ${ }^{1} \mathrm{~L}_{\mathrm{b}}\left(\pi \pi^{*}\right)$ levels studied here. This is confirmed by our measurements on 9-methyladenine, a molecule in which the $\mathrm{N}_{9}-\mathrm{H}$ dissociation channel is closed. Moreover, we find that the linewidths of the $M_{1}-M_{4}$ transitions qualitatively follow the trends observed for their counterparts $A_{1}-A_{4}$.

As far as possible candidates for fast decay pathways of low-lying ${ }^{1} \mathrm{n} \pi^{*}$ and ${ }^{1} \mathrm{~L}_{\mathrm{b}}\left(\pi \pi^{*}\right)$ levels are concerned, ab initio calculations agree that the main decay channel of the ${ }^{1} \mathrm{~L}_{\mathrm{b}}\left(\pi \pi^{*}\right)$ state involves internal conversion to the ${ }^{1} \mathrm{n} \pi^{*}$ state via a conical intersection between these two states. ${ }^{5,27}$ The calculations predict that the ${ }^{1} \mathrm{n} \pi^{*} /{ }^{1} \mathrm{~L}_{\mathrm{b}}\left(\pi \pi^{*}\right)$ conical intersection lies between 0.05 and $0.14 \mathrm{eV}$ above the ${ }^{1} \mathrm{~L}_{\mathrm{b}}\left(\pi \pi^{*}\right)$ minimum, depending on the finer details of the calculations, with no barrier between the ${ }^{1} \mathrm{~L}_{\mathrm{b}}\left(\pi \pi^{*}\right)$ minimum and the conical intersection. Other possible decay pathways that have been considered involve radiative decay to the ground state, non-radiative internal conversion to the ground state, and intersystem crossing to the triplet manifold, but these are thought to be of no or minor importance. ${ }^{5}$ For the ${ }^{1} \mathrm{n} \pi^{*}$ state, on the other hand, it has been concluded that its decay is not governed by a conical intersection with the ground state but rather by intersystem crossing to the isoenergetic $T_{1}\left(\pi \pi^{*}\right)$ state. ${ }^{5,11,29}$ These considerations lead us to the conclusion that the large increase of the linewidth of the vibrationless transitions to the ${ }^{1} \mathrm{~L}_{\mathrm{b}}\left(\pi \pi^{*}\right)$ state of adenine and 9-methyladenine in the helium environment should be ascribed to a dramatically increased rate for internal conversion rate via the ${ }^{1} \mathrm{n} \pi^{*} /{ }^{1} \mathrm{~L}_{\mathrm{b}}\left(\pi \pi^{*}\right)$ conical intersection.

Normally, it is assumed that liquid helium droplets provide a matrix in which the effects of the surroundings on the dopant are minimal. While the observed transition frequencies support this assumption, the large variations in the increase of the linewidths are clearly at odds with this assumption. It should be noted that effects of the helium matrix as observed in the present study are not unprecedented. For example, studies on benzo[ $g, h, i]$ perylene show that in a helium environment the $S_{2}$ band origin is significantly broadened. ${ }^{73}$ This has been attributed to an enhanced $\mathrm{S}_{2} \rightarrow \mathrm{S}_{1}$ internal conversion in liquid helium. Even more striking are the effects on the photochemistry of 3-hydroxyflavone. ${ }^{74}$ Here it was found that excited state intramolecular proton transfer is promoted by the helium environment to such an extent that it becomes a barrierless process.

In the present study, the helium induced shifts of the transition frequencies are comparable for the ${ }^{1} \mathrm{n} \pi^{*}$ and ${ }^{1} \mathrm{~L}_{\mathrm{b}}\left(\pi \pi^{*}\right)$ states, which indicates that at their equilibrium geometries both states are affected in a similar way by the helium environment. This does not necessarily imply that this also holds for the geometry of the ${ }^{1} \mathrm{n} \pi * /{ }^{1} \mathrm{~L}_{\mathrm{b}}\left(\pi \pi^{*}\right)$ conical intersection. As a matter of fact, this geometry resembles to some extent the equilibrium geometry of the ${ }^{1} \mathrm{~L}_{b}\left(\pi \pi^{*}\right)$ state ${ }^{27,30}$ whereas the equilibrium geometry of the ${ }^{1} \mathrm{n} \pi^{*}$ is significantly different. As a result, the helium environment very likely affects both states to a different extent. This could lead to a lowering of energy of the conical intersection and hence cause an increase of the relaxation rate for the ${ }^{1} \mathrm{~L}_{b}\left(\pi \pi^{*}\right)$ state.

As mentioned previously, only a few resonances have been assigned in the excitation spectrum of adenine and 9-methyladenine. Here we find that ${ }^{1} \mathrm{n} \pi *$ vibronic levels of overall $\mathrm{A}^{\prime \prime}$ symmetry (for example, $A_{1}$ ) have a relatively long lifetime, while ${ }^{1} \mathrm{~L}_{\mathrm{b}}\left(\pi \pi^{*}\right)$ levels and ${ }^{1} \mathrm{n} \pi^{*}$ vibronic levels of overall $\mathrm{A}^{\prime}$ symmetry, which are vibronically coupled to the ${ }^{1} \mathrm{~L}_{\mathrm{b}}\left(\pi \pi^{*}\right)$ state (for example, $A_{2}$ and $A_{4}$ ), have a significantly reduced lifetime. Under the reasonable assumption that the lifetime of ${ }^{1} \mathrm{~L}_{\mathrm{b}}\left(\pi \pi^{*}\right)$ vibronic levels in helium nanodroplets is shorter than that of its vibrationless level, our results indicate that none of the $A_{3}-A_{6}$ resonances are associated with the ${ }^{1} \mathrm{~L}_{\mathrm{b}}\left(\pi \pi^{*}\right)$ state. Rather, these resonances correspond to levels that are vibronically coupled to the ${ }^{1} \mathrm{~L}_{\mathrm{b}}\left(\pi \pi^{*}\right)$ state. The observed propensity of ${ }^{1} \mathrm{n} \pi^{*}$ vibronic states of $\mathrm{A}^{\prime \prime}$ symmetry having a relatively long and of $\mathrm{A}^{\prime}$ symmetry a relatively short lifetime suggests that the $A_{3}$ and $A_{5}$ transitions are associated with ${ }^{1} \mathrm{n} \pi^{*}\left(\mathrm{~A}^{\prime}\right)$ vibronic levels while resonance $A_{6}$ most probably results from excitation of a ${ }^{1} n \pi^{*}\left(\mathrm{~A}^{\prime \prime}\right)$ vibronic level.

\section{Conclusions}

The application of absorption spectroscopy under helium nanodroplet conditions has enabled us to elucidate the spectroscopy and dynamics of electronically excited states of adenine, 9-methyladenine and 2-aminopurine. Our studies have shown that the spectroscopy of these compounds is not affected by the helium environment in other ways than what has been observed for other molecules. However, at the same time we have found that the excited-state dynamics are much more susceptible to the helium environment than what intuitively would have been expected. This is evidenced by the resonances in the absorption spectra of adenine and 9-methyladenine that show a surprisingly large variation in linewidth. In combination with previous assignments of the resonances, it has been argued that these large variations are in line with the conclusions of theoretical calculations that predict that decay to the ${ }^{1} n \pi^{*}$ state is the predominant pathway for decay of the ${ }^{1} \mathrm{~L}_{\mathrm{b}}\left(\pi \pi^{*}\right)$ state. The observed enhancement of the ${ }^{1} \mathrm{~L}_{\mathrm{b}}\left(\pi \pi^{*}\right)$ decay rate is then due to a lowering of the conical intersection between the two states in helium nanodroplets. The $\mathrm{S}_{0} \rightarrow \mathrm{S}_{1}$ absorption spectrum obtained for 2-aminopurine fully confirms these conclusions. In this molecule $\mathrm{S}_{1}$ is the ${ }^{1} \mathrm{~L}_{\mathrm{a}}\left(\pi \pi^{*}\right)$ state that has no low-lying conical intersections with the electronic ground state or other electronically excited states. Accordingly, the $0-0$ transition is narrow, and higher-lying resonances only show limited broadening due to enhanced vibrational relaxation.

So far, the influence of the helium environment on the properties of electronically excited states of embedded molecules has been restricted to consideration of properties of potential energy surfaces close to equilibrium. The present study in combination with other studies now clearly starts to indicate that such a description may not be appropriate further away from equilibrium. We therefore hope that the present results will provide impetus for further theoretical studies on helium-solute interactions in these regions. 


\section{Acknowledgements}

This work was supported by the Swiss National Science Foundation through Grant No. 200020-119789, the "Stichting voor Fundamenteel Onderzoek der Materie (FOM)", and The Netherlands Organization for Scientific Research (NWO). AMR acknowledges the Netherlands Organization for Scientific Research (NWO) for a VENI post-doctoral fellowship.

\section{References}

1 B. Cohen, P. M. Hare and B. Kohler, J. Am. Chem. Soc., 2003, 125, 13594.

2 C. T. Middleton, K. de La Harpe, C. Su, Y. K. Law, C. E. Crespo-Hernandez and B. Kohler, Annu. Rev. Phys. Chem., 2009, 60, 217.

3 C. Z. Bisgaard, H. Satzger, S. Ullrich and A. Stolow, ChemPhysChem, 2009, 10, 101.

4 H. Saigusa, J. Photochem. Photobiol., C, 2006, 7, 197.

5 I. Conti, M. Garavelli and G. Orlandi, J. Am. Chem. Soc., 2009, 131, 16108.

6 J. R. Platt, J. Chem. Phys., 1949, 17, 484.

7 L. B. Clark, G. G. Peschel and I. Tinoco, J. Phys. Chem., 1965, 69, 3615 .

8 D. Voet, W. B. Gratzer, R. A. Cox and P. Doty, Biopolymers, 1963, 1, 193.

9 L. B. Clark and I. Tinoco, J. Am. Chem. Soc., 1965, 87, 11.

10 E. Nir, K. Kleinermanns, L. Grace and M. S. de Vries, J. Phys. Chem. A, 2001, 105, 5106.

11 D. C. Luhrs, J. Viallon and I. Fischer, Phys. Chem. Chem. Phys., 2001, 3, 1827

12 N. J. Kim, G. Jeong, Y. S. Kim, J. Sung, S. K. Kim and Y. D. Park, J. Chem. Phys., 2000, 113, 10051.

13 N. J. Kim, H. Kang, Y. D. Park and S. K. Kim, Phys. Chem. Chem. Phys., 2004, 6, 2802.

14 Y. Lee, M. Schmitt, K. Kleinermanns and B. Kim, J. Phys. Chem. A, 2006, 110, 11819.

15 C. Plutzer and K. Kleinermanns, Phys. Chem. Chem. Phys., 2002, 4, 4877.

16 C. Plutzer, E. Nir, M. S. de Vries and K. Kleinermanns, Phys. Chem. Chem. Phys., 2001, 3, 5466.

17 T. Pancur, N. K. Schwalb, F. Renth and F. Temps, Chem. Phys., 2005, 313, 199.

18 H. Satzger, D. Townsend and A. Stolow, Chem. Phys. Lett., 2006, 430, 144.

19 E. Samoylova, H. Lippert, S. Ullrich, I. V. Hertel, W. Radloff and T. Schultz, J. Am. Chem. Soc., 2005, 127, 1782.

20 H. Kang, B. Jung and S. K. Kim, J. Chem. Phys., 2003, 118, 6717.

21 S. Ullrich, T. Schultz, M. Z. Zgierski and A. Stolow, J. Am. Chem. Soc., 2004, 126, 2262.

22 H. Kang, K. T. Lee, B. Jung, Y. J. Ko and S. K. Kim, J. Am. Chem. Soc., 2002, 124, 12958.

23 C. Canuel, M. Mons, F. Piuzzi, B. Tardivel, I. Dimicoli and M. Elhanine, J. Chem. Phys., 2005, 122, 074316.

24 S. Ullrich, T. Schultz, M. Z. Zgierski and A. Stolow, Phys. Chem. Chem. Phys., 2004, 6, 2796.

25 A. Broo, J. Phys. Chem. A, 1998, 102, 526.

26 M. Z. Zgierski, S. Patchkovskii and E. C. Lim, Can. J. Chem., $2007, \mathbf{8 5}, 124$.

27 L. Serrano-Andres, M. Merchan and A. C. Borin, Chem.-Eur. J., 2006, 12, 6559 .

28 C. M. Marian, J. Chem. Phys., 2005, 122, 104314.

29 L. Blancafort, J. Am. Chem. Soc., 2006, 128, 210.

30 S. Perun, A. L. Sobolewski and W. Domcke, J. Am. Chem. Soc., 2005, 127, 6257.

31 W. M. I. Hassan, W. C. Chung, N. Shimakura, S. Koseki, H. Kono and Y. Fujimura, Phys. Chem. Chem. Phys., 2010, 12, 5317.

32 A. L. Sobolewski, W. Domcke, C. Dedonder-Lardeux and C. Jouvet, Phys. Chem. Chem. Phys., 2002, 4, 1093.
33 A. L. Sobolewski and W. Domcke, Eur. Phys. J. D, 2002, 20, 369.

34 S. Perun, A. L. Sobolewski and W. Domcke, Chem. Phys., 2005, 313, 107.

35 J. P. Toennies and A. F. Vilesov, Angew. Chem., Int. Ed., 2004, 43, 2622.

36 F. Stienkemeier and A. F. Vilesov, J. Chem. Phys., 2001, 115, 10119.

37 M. Y. Choi, G. E. Douberly, T. M. Falconer, W. K. Lewis, C. M. Lindsay, J. M. Merritt, P. L. Stiles and R. E. Miller, Int. Rev. Phys. Chem., 2006, 25, 15.

38 F. Stienkemeier and K. K. Lehmann, J. Phys. B: At., Mol. Opt. Phys., 2006, 39, R127.

39 M. Wewer and F. Stienkemeier, Phys. Rev. B: Condens. Matter, 2003, 67, 125201 .

40 S. Smolarek, A. M. Rijs, J. S. Hannam, D. A. Leigh, M. Drabbels and W. J. Buma, J. Am. Chem. Soc., 2009, 131, 12902.

41 M. Wewer and F. Stienkemeier, J. Chem. Phys., 2004, 120, 1239.

42 M. Wewer and F. Stienkemeier, Phys. Chem. Chem. Phys., 2005, 7, 1171.

43 J. P. Toennies, A. F. Vilesov and K. B. Whaley, Phys. Today, 2001, 54, 31.

44 E. Loginov, A. Braun and M. Drabbels, Phys. Chem. Chem. Phys., 2008, 10, 6107.

45 E. Loginov and M. Drabbels, J. Phys. Chem. A, 2007, 111, 7504.

46 A. Braun and M. Drabbels, J. Chem. Phys., 2007, 127, 114303.

47 S. Smolarek, A. Vdovin, D. L. Perrier, J. P. Smit, M. Drabbels and W. J. Buma, J. Am. Chem. Soc., 2010, 132, 6315.

48 M. Hartmann, F. Mielke, J. P. Toennies, A. F. Vilesov and G. Benedek, Phys. Rev. Lett., 1996, 76, 4560.

49 R. Schmied, P. Carcabal, A. M. Dokter, V. P. A. Lonij, K. K. Lehmann and G. Scoles, J. Chem. Phys., 2004, 121, 2701.

50 E. Nir, C. Plutzer, K. Kleinermanns and M. de Vries, Eur. Phys. J. D, 2002, 20, 317.

51 K. A. Seefeld, C. Plutzer, D. Lowenich, T. Haber, R. Linder, K. Kleinermanns, J. Tatchen and C. M. Marian, Phys. Chem. Chem. Phys., 2005, 7, 3021.

52 M. Hartmann, A. Lindinger, J. P. Toennies and A. F. Vilesov, J. Phys. Chem. A, 2001, 105, 6369.

53 R. Lehnig and A. Slenczka, J. Chem. Phys., 2005, 122, 244317.

54 R. Lehnig and A. Slenczka, J. Chem. Phys., 2003, 118, 8256.

55 E. Loginov, D. Rossi and M. Drabbels, Phys. Rev. Lett., 2005, 95, 163401.

56 P. Radcliffe, A. Przystawik, T. Diederich, T. Doppner, J. Tiggesbaumker and K. H. Meiwes-Broer, Phys. Rev. Lett., 2004, 92, 173403.

57 K. Feng, G. Engler, K. Seefeld and K. Kleinermanns, ChemPhysChem, 2009, 10, 886.

58 L. Serrano-Andres, M. Merchan and A. C. Borin, Proc. Natl. Acad. Sci. U. S. A., 2006, 103, 8691.

59 A. C. Borin, L. Serrano-Andres, V. Ludwig, K. Coutinho and S. Canuto, Int. J. Quantum Chem., 2006, 106, 2564.

60 S. Perun, A. L. Sobolewski and W. Domcke, Mol. Phys., 2006, 104, 1113.

61 S. K. Mishra, M. K. Shukla and P. C. Mishra, Spectrochim. Acta, Part A, 2000, 56, 1355.

62 M. Y. Choi, F. Dong, S. W. Han and R. E. Miller, J. Phys. Chem. A, 2008, 112, 7185 .

63 Until recently, it was assumed that the transition at $35497 \mathrm{~cm}^{-1}$ in the gas phase corresponded to the $0-0$ transition to the ${ }^{1} n \pi^{*}$ state. Recent computational studies of the vibronic activities associated with the transition to this state suggest, however, that the band is associated with excitation of the $v=6$ level of the anharmonic puckering coordinate. $^{64}$ This would imply that also the $36063 \mathrm{~cm}^{-1}$ transition involves excitation of another vibronic level of the ${ }^{1} n \pi^{*}$ state than previously thought.

64 I. Conti, E. Di Donato, F. Negri and G. Orlandi, J. Phys. Chem. A, 2009, 113, 15265 .

65 A. Lindinger, E. Lugovoj, J. P. Toennies and A. F. Vilesov, Z. Phys. Chem. (Munich), 2001, 215, 401.

66 O. Birer, P. Moreschini, K. K. Lehmann and G. Scoles, J. Phys. Chem. A, 2007, 111, 12200. 
67 O. Birer, P. Moreschini, K. K. Lehmann and G. Scoles, J. Phys. Chem. A, 2007, 111, 7624.

68 Two-photon ionization to the $\mathrm{D}_{1}\left(\mathrm{n}^{-1}\right)$ state is energetically not possible in the investigated excitation energy region. As a result, the ionization cross section via the ${ }^{1} \mathrm{n} \pi^{*}$ state can be expected to be small. This most likely is the reason that also via the $A_{1}$ transition no resonance enhanced two-photon ionization signal has been observed.

69 S. Peng, A. Padva and P. R. Lebreton, Proc. Natl. Acad. Sci. U. S. A., 1976, 73, 2966.
70 H. Satzger, D. Townsend, M. Z. Zgierski, S. Patchkovskii, S. Ullrich and A. Stolow, Proc. Natl. Acad. Sci. U. S. A., 2006, 103, 10196.

71 M. G. D. Nix, A. L. Devine, B. Cronin and M. N. R. Ashfold, J. Chem. Phys., 2007, 126, 124312.

72 S. B. Nielsen and T. I. Solling, ChemPhysChem, 2005, 6, 1276.

73 O. Birer, P. Moreschini and K. K. Lehmann, Phys. Chem. Chem. Phys., 2008, 10, 1648.

74 R. Lehnig, D. Pentlehner, A. Vdovin, B. Dick and A. Slenczka, J. Chem. Phys., 2009, 131, 194307. 